04

\title{
Средняя скорость электронов в области катодного падения при азотировании в тлеющем разряде
}

\author{
(C) И.М. Пастух, В.В. Люховець \\ Хмельницкий национальный университет, \\ 28016 Хмельницкий, Украина \\ e-mail: pastim@mail.ru
}

(Поступило в Редакцию 18 июля 2016 г.)

Теоретически решена и экспериментально проверена задача о средней скорости электронов в области катодного падения тлеющего разряда при азотировании. Приведены методика и аналитический аппарат для практического расчета средней скорости электронов.

DOI: 10.21883/JTF.2017.04.44309.1999

\section{Введение}

Рассмотренная ранее [1] модель газоразрядных процессов при азотировании в тлеющем разряде базируется на учете реально значимых субпроцессов [2], частота которых в общем виде в основном зависит от средней относительной скорости движения инициатора и контрчастицы процесса. Для подавляющего большинства реально присутствующих субпроцессов (с учетом энергетики вакуумно-диффузионных газоразрядных технологий (ВДГРТ), к которым относится азотирование в тлеющем разряде, и состава газовых сред, применяемых в нем) инициатором выступают электроны. Их скорость несравненно больше скорости контрчастиц столкновения, что позволяет считать последние условно неподвижными, поэтому задача о средней скорости относительного движения электронов и частиц сводится к определению средней скорости электронов.

Принято считать, что вероятность скорости электронов $V_{e x}$ в любом слое одномерной области катодного падения (ОКП) с координатой $x$ подчиняется распределению Максвелла $M(V)$, которое в незначительной степени может деформироваться эффектами второго порядка [3] (далее для упрощения выражений индексы причастности параметра к электрону и координате слоя не указываются):

$$
P(V, x)=M(V) d V=C_{M} \frac{V^{2}}{c^{3}} e^{-\frac{V^{2}}{c^{2}}} d V,
$$

где $P(V, x)$ - вероятность наличия в слое на расстоянии от катода $x$ электронов со скоростью в диапазоне $V \ldots V+d V, c-$ наиболее вероятная скорость электронов в слое ОКП с координатой $x, C_{M}-$ константа нормирования распределения на единицу, $C_{M}=4 / \sqrt{\pi}$.

Одномерная модель допустима, если учесть особенности конструктивной схемы, взаимно охватывающей системы анод-катод, характерной для процессов ВДГРТ.

Известны также исследования, в которых приводятся ссылки на другие распределения, например на распределение Druyvesteyn M.I. [3-5], которое отличается в основном тем, что скорости входят в показатель экспоненты в четвертой степени. В этом случае так называемый „хвост“ распределения, соответствующий большим значениям энергии, убывает более интенсивно в сравнении с распределением $M(V)$, что несущественно для решаемой задачи с учетом энергетических уровней, характерных для ВДГРТ. Кроме того, для изложенной ниже методики решения задачи о средней скорости вид распределения вероятностей принципиальной роли не играет, поскольку не меняет принятой в ней модели.

Известны методы решения задачи о скорости электронов [3], основанные на кинетическом уравнении для электронов как частного случая общего кинетического уравнения Больцмана для функции распределения частиц в газовой среде. Недостатком метода является, прежде всего, его сложность (возможно только приближенное решение, например, на основе системы полиномов Лежандра, так называемое приближение Лоренца, которое может применяться только для несильных полей, что исключает возможность его использования в ОКП ВДГРТ) [6].

\section{Теоретические предположения при определении средней скорости электронов}

Предлагаемая методика базируется на применении для каждого слоя с координатой $x$ понятия базовой скорости $V_{x}$. В качестве базовой принята скорость, которую имеет электрон, прошедший весь путь от катода к слою $x$ без столкновений. За пределами ширины части ОКП с гарантированной идентификацией базовой скорости средняя скорость может определяться экстраполяцией закона изменения ее в этой части на диапазон, где такая идентификация по предложенной методике базовой скорости невозможна. Следует заметить, что некоторая погрешность в этом случае несравненно меньше в сопоставлении с расчетами, в которых сечение субпроцессов с целью упрощения не зависит от скорости электронов и принимается постоянной. 
При линейном законе изменения напряженности поля в ОКП базовая скорость определяется

$$
V_{x}=\sqrt{\frac{2 \varepsilon_{x}}{m_{e}}}=\sqrt{\frac{2 \varphi_{x} q_{e}}{m_{e}}}=\sqrt{\frac{4 U_{K}\left(x-x^{2} / 2 \delta\right) q_{e}}{\delta m_{e}}},
$$

где $\varepsilon_{x}$ - энергия электрона, который двигался без столкновений от катода к слою $x, m_{e}$ - масса электрона, $\varphi_{x}$ - потенциал поля в слое с координатой $x$.

Вероятность столкновения электрона с произвольной контрчастицей сорта $W$ на пути от катода к точке ОКП с координатой $x$

$$
P_{C}=Q_{C} / Q,
$$

где $Q_{C}$ - эффективный объем пространства возможного столкновения в объеме газа $Q=s x$ с основанием размером в единичную площадь $s$ и длиной $x$, $Q_{C}=s_{c} x=\left(s_{c n}+s_{c q}\right) x, s_{c}, s_{c n}, s_{c q}-$ сумма эффективных площадей мишеней столкновений, в том числе $s$ соответственно с нейтральными и заряженными частицами.

$$
\begin{aligned}
s_{c n} & =\sum_{i=1}^{W} z_{W i} \sigma_{c i}=\sum_{i=1}^{W} n_{W i} Q \sigma_{c i} \\
& =x s \sum_{i=1}^{W} C_{p} p_{W i} \sigma_{c i}=C_{p} x s p \sum_{i=1}^{W} K_{p W_{i}} \sigma_{c i},
\end{aligned}
$$

где $z_{W i}$ - количество частиц произвольного сорта в объеме газа $Q, \sigma_{c i}$ - эффективное сечение столкновений, которое определяется через эффективный диаметр контрчастицы, при этом размерами самого электрона пренебрегаем, $\sigma_{c i}=\pi d_{W i}^{2} / 4, n_{W i}-$ концентрация частиц произвольного сорта, $p_{W i}$ - парциальное давление произвольного компонента, $C_{P}-$ константа концентрации компонента, $C_{P}=1 / k T, k-$ константа Больцмана, $T-$ температура по шкале Кельвина, $K_{p W}$ - коэффициент объемной доли компонента в газовой среде.

$$
s_{c q} \int_{0}^{x} n_{q}(x) \sigma_{c q}(V) d x
$$

где $n_{q}(x)$ - концентрация заряженных частиц в зависимости от координаты поля, $\sigma_{c q}(V)-$ сечение столкновений электрона с заряженной частицей.

Следует заметить, что закон распределения заряженных частиц $n_{q}(x)$ может быть определен по методике, для которой нужно среднее значение скорости электронов, но это как раз и является целью настоящей работы. Поэтому для допустимого упрощения эта составляющая не учитывается, при этом примем во внимание, что при сравнительно невысокой степени ионизации частота рекомбинационных явлений и электрон-электронных столкновений чрезвычайно мала. Кроме того, потеря этой составляющей компенсируется тем, что при расчете суммы эффективных площадей мишеней столкновений с нейтральными частицами не учитывается то, что

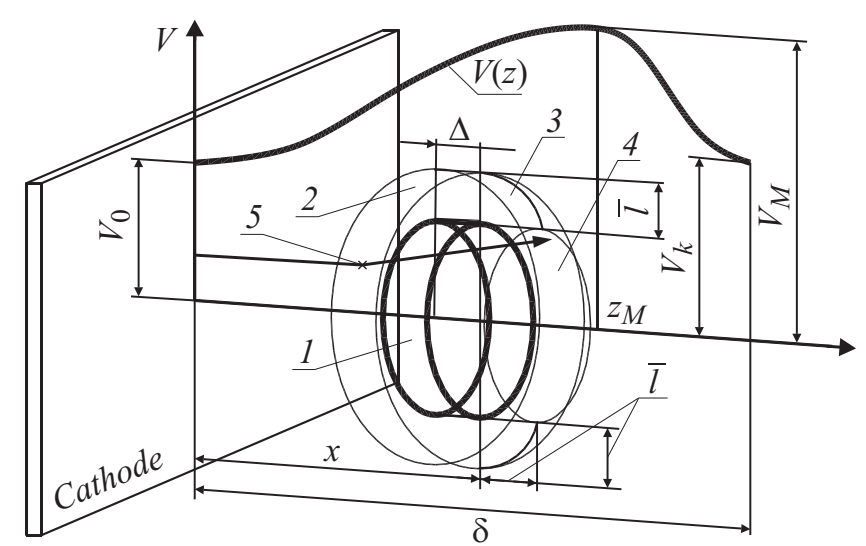

Рис. 1. Пространственная схема к расчету средней скорости электронов.

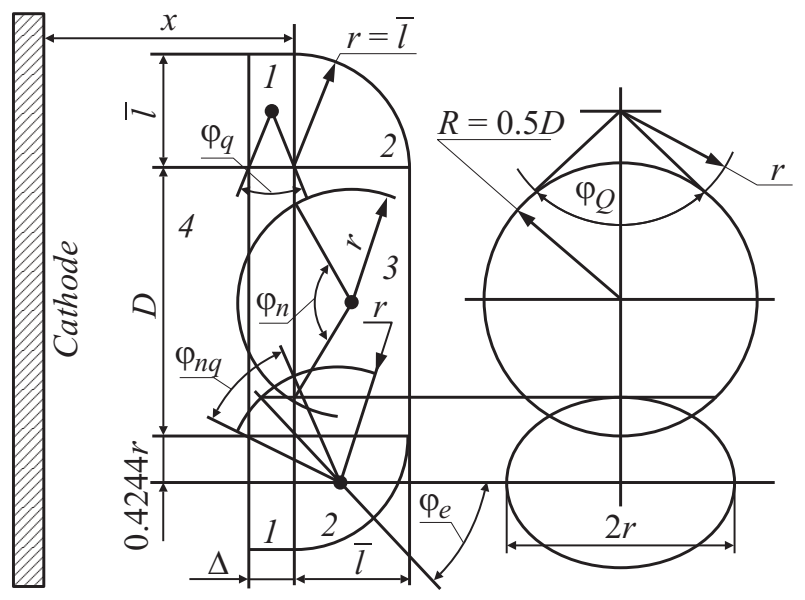

Рис. 2. Схема формирования контрольного объема (зоны отражения: 1 - бокового, 2 - переднебокового, 3 - переднего, 4 - вторичного).

некоторые из них частично или даже в полной мере взаимно перекрываются, т.е. сумма площадей в действительности несколько меньше. С этими допущениями вероятность столкновения электрона

$$
P_{C}=x C_{p} p \sum_{i=1}^{W} K_{p W_{i}} \sigma_{c i}=x C_{p} p \sigma_{v c}
$$

где $\sigma_{v c}=\sum_{i=1}^{W} K_{p W_{i}} \sigma_{c i}-$ эквивалентное сечение упругих столкновений электрона с компонентами газовой среды.

Вероятность бесстолкновительного пробега электрона от катода к слою с координатой $x$ есть $P_{0}=1-P_{C}$.

Для определения параметров распределения вероятностей скоростей электронов в слое предлагается модель опорной точки. Пространственная схема этой модели иллюстрируется рис. 1, схема формирования контрольного объема - рис. 2, схема определения средней скорости - рис. 3.

Базовую скорость $V_{x}$ будут иметь не только электроны, которые без столкновений прошли путь от 


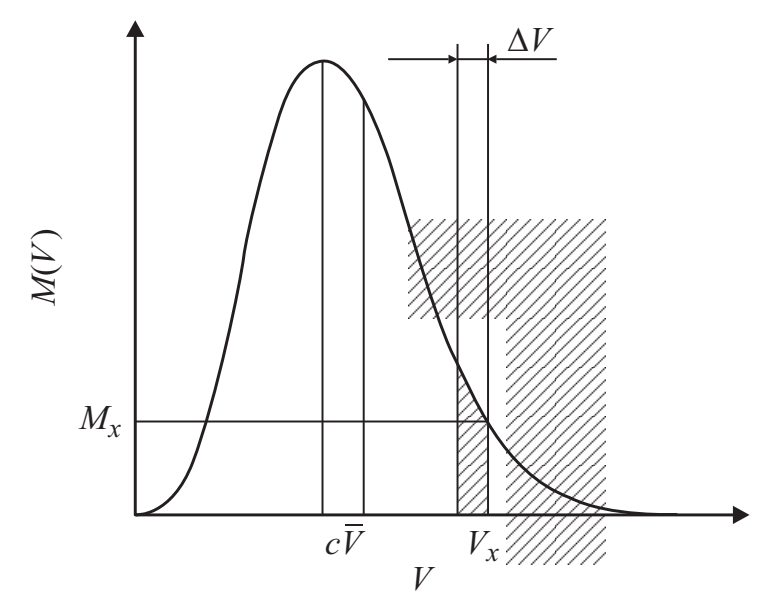

Рис. 3. Схема определения средней скорости электронов.

катода к слою с координатой $x$. Кроме этих электронов, примерно такую же скорость будут иметь и электроны, которые вернулись в слой $x$ после отражения их в результате столкновения в некотором элементе фазового пространства перемещений - контрольном объеме (КО). Размеры этого объема устанавливаются исходя из следующих соображений. Очевидно, что для удобства вычислений с использованием плотности тех или иных потоков целесообразно основу КО выбрать в форме круга (минимальный периметр) с единичной площадью $s$. Тогда диаметр круга определится как $D=\sqrt{4 s / \pi}$. Толщина контрольного объема $\Delta$ может быть принята, если одновременно с ней определиться с диапазоном скоростей $\Delta V$. Возвращение электрона в ту же координату поля $x$ после отражения обеспечило бы ему тот же базовый энергетический уровень $\varepsilon_{x}$, если бы не было потерь энергии. Итак, электрон, который сначала пролетел от катода через слой $x$ без столкновений, вернется в результате упругого столкновения (неупругие столкновения не рассматриваются, поскольку они существенно уменьшают энергию электрона, и она становится несравненно меньше по сравнению с базовой) в эту же координату поля с энергией, которая уменьшится на величину

$$
\Delta \varepsilon=\varepsilon_{x} \frac{2 m_{e}}{m_{v}}(1-\cos \theta)=\frac{m_{e}^{2} V_{x}^{2}}{m_{v}},
$$

где $m_{v}$ - приведенная масса контрчастиц столкновений, $m_{v}=\sum_{i=1}^{W} K_{p W_{i}} m_{W i}, m_{w i}-$ масса контрчастиц соответствующего сорта, $\theta$ - угол изменения направления движения.

Так как схема столкновения считается сферически симметричной, можно для упрощения не учитывать составляющую $(1-\cos \theta)$.

Через диапазон уменьшения энергии можно определить диапазон скоростей электрона, в рамках которого отслеживается вероятность его кинетических параметров $\Delta V=V_{x}-\sqrt{V_{x}^{2}-\frac{2 \Delta \varepsilon}{m_{e}}}$. Поскольку диапазону потерь энергии соответствует определенный диапазон изменения потенциала поля, из уравнения

$$
\begin{aligned}
\Delta \varphi & =\frac{\Delta \varepsilon}{q_{e}}=\frac{2 U_{K}}{\delta}\left[\left(x-\frac{x^{2}}{2 \delta}\right)-(x-\Delta)+\frac{(x-\Delta)^{2}}{2 \delta}\right] \\
& =\frac{U_{K}}{\delta^{2}}\left[\Delta^{2}+2(\delta-x) \Delta\right]
\end{aligned}
$$

можно определить размер толщины КО $\Delta$, второй корень уравнения (со знаком минус перед всеми составляющими) не имеет смысла $\left(\delta\right.$ - ширина ОКП, $q_{e}-$ заряд электрона). Замена бесконечно малой ширины диапазона скоростей $d V$ конечным $\Delta V$ заставляет в дальнейшем оперировать понятием не полной вероятности, а статистической вероятностью, эта замена вносит некоторую неточность, однако величина ее не играет существенной роли. Например, для газовой смеси, состоящей по объему из 75\% $\mathrm{N}_{2}$ и $25 \% \mathrm{Ar}$ (смесь $\mathrm{N} 75 \mathrm{Ar} 25$, здесь и далее все газовые среды обозначаются по аналогичному принципу), отношение диапазона скорости к скорости составляет $K_{V}=\Delta V / V=0.0000177$, а вообще для газов, которые реально применяются в ВДГРТ, $K_{V}=0.0000124 \ldots 0.0000321$ (соответственно для углекислого газа и аммиака). Отражение электронов бесстолкновительного движения в КО возможно как первичное, так и вторичное и все последующие, но поскольку вероятность последовательно следующих и зависимых процессов определяется как произведение составляющих вероятностей, то вторичные и последующие процессы учитываться не будут в связи с их исчезающе малой величиной. Действительно, для того чтобы в КО попал электрон в результате вторичного отражения, необходимо учесть вероятность бесстолкновительного движения в одну из областей $1-3$ (рис. 2), отражения в область 4 и последующего отражения в КО. При этом нужно было бы значительно расширить размер ширины диапазона скоростей $\Delta V$, поскольку потери энергии в результате двух столкновений удвоились бы, а это внесло бы заметную погрешность (форма заштрихованной зоны - трапециевидная). Первичное отражение возможно с передней 3, переднебоковой 2 и боковой 1 зон. Размеры этих зон регламентируются соответствующими размерами КО и средней длиной свободного пробега электронов в газовой смеси. Вероятность попадания электронов в КО в результате первичного отражения из любой зоны $Z$

$$
P_{B}(Z)=P_{0}(Z) P_{C}(Z) P_{B \varphi}(Z)
$$

где $P_{0}(Z)$ - вероятность бесстолкновительного пробега электрона в зону $Z, P_{C}(Z)$ - вероятность упругого столкновения в этой зоне, $P_{B \varphi}(Z)$ - вероятность отражения электрона из зоны $Z$ в контрольный объем.

Величина телесного угла $\varphi$ зависит от положения точки столкновения в конкретной области. Очевидно, что среднее его значение, определенное путем интегрирования в пределах зоны, будет соответствовать 
значению угла для положения точки столкновения в центре масс фигуры зоны. Поэтому все дальнейшие выкладки относительно показателей вероятности модели относятся именно к точкам, которые являются центрами масс соответствующих зон. По аналогии с формулой (6) вероятности бесстолкновительного пробега в соответствующие зоны определятся

$$
\begin{gathered}
P_{0}(1)=1-(x-0.5 \Delta) C_{p} p \sigma_{v c}, \\
P_{0}(2)=1-(x+0.424 \bar{l}) C_{p} p \sigma_{v c}, \\
P_{0}(3)=1-(x+0.5 \bar{l}) C_{p} p \sigma_{v c} .
\end{gathered}
$$

Вероятность упругих столкновений электронов с контрчастицами в любой зоне

$$
P_{C}(Z)=s_{C Z} / s_{Z}
$$

где $s_{C Z}-$ суммарная площадь мишеней столкновений в зонах, $s_{Z}$ - площадь зоны в сечении, перпендикулярном оси поля.

Для каждой зоны при многокомпонентном составе газовой смеси суммарная площадь мишеней определяется как произведение количества частиц соответствующего сорта $z_{W i}$ в объеме зоны $Q_{Z}$, концентрация которых $n_{W i}$, на сечение упругих столкновений $\sigma_{c W i}$ :

$$
\begin{aligned}
s_{C Z} & =z_{W i} \sigma_{C W i}=n_{W i} \sigma_{C W i} Q_{Z} \\
& =p C_{p} s_{Z} x_{Z} \sum_{i=1}^{W} K_{p W i} \sigma_{c W i}=p C_{p} s_{Z} x_{Z} \sigma_{v c} .
\end{aligned}
$$

Тогда вероятность столкновений в любой зоне $P_{C}(Z)=p C_{p} \sigma_{v c} s_{Z} x_{Z} / s_{Z}$, где $x_{Z}-$ средние протяженности соответствующих зон из рис. 2 определяются: $x_{1}=\Delta, x_{2}=\pi \bar{l} / 4, x_{3}=\bar{l} ; p-$ давление газовой среды. Площади соответствующих зон из того же рисунка $s_{1}=s_{2}=4 \pi\left(R \bar{l}+\bar{l}^{2}\right), s_{3}=\pi R^{2}$. Вероятность отражения электрона из зоны $Z$ в контрольный объем должна учитывать не только отношение объемов коноида отражения в КО и полной сферы, но и то обстоятельство, что при равновероятности всех прицельных параметров $\rho$ угол изменения направления движения $\theta$ неравновероятен, поскольку зависимость между прицельным параметром и углом отражения нелинейная. Таким образом, вероятность отражения в КО

$$
P_{B \varphi}(Z)=\bar{K}_{\theta} P_{B}(\varphi)
$$

где $\bar{K}_{\theta}-$ среднее значение коэффициента коррекции вероятности в зависимости от угла $\theta$ изменения направления движения электрона после отражения, $P_{B}(\varphi)-$ вероятность отражения электрона из зоны $Z$ в сектор $\varphi$.

Коэффициент коррекции вероятности выбирается, исходя из анализа интенсивности изменения угла $\theta$ при равномерном изменении прицельного параметра $\rho$, которая характеризуется производной при единичном радиусе контрчастицы $0.5 d_{W i}$. $d \theta / d \rho=d(2 \arccos (\rho)) / d \rho=2 / \sqrt{1-\rho^{2}}$. Наименьшая интенсивность изменения угла будет иметь место, когда $\theta=\pi$, поэтому при этом значении угла коэффициент коррекции нормируется на единицу. Отношение производной при $\theta=\pi$ к производной для произвольного угла дает коэффициент коррекции $K_{\theta}=\sqrt{1-\rho^{2}}=\sqrt{1-\cos ^{2}(\theta / 2)}=\sin (\theta / 2)$. Среднее значение коэффициента коррекции в диапазоне углов изменения направления движения электрона в результате столкновения от начального $\theta_{\Pi}$ к конечному $\theta_{K}$ (с учетом того, что отсчет углов ведется при сферической симметрии модели отражение в направлении, противоположном оси поля, а $\theta_{K}-\theta_{\Pi}=\varphi$ )

$$
\bar{K}_{\theta}=\frac{\int_{\theta_{\Pi}}^{\theta_{K}} \sin \frac{\theta}{2} d \theta}{\theta_{K}-\theta_{\Pi}}=\frac{2\left(\cos \frac{\theta_{\Pi}}{2}-\cos \frac{\theta_{K}}{2}\right)}{\varphi} .
$$

Заметим, что формула (14) может использоваться только в диапазоне углов от нуля до $\pi$, т. е. для случая, когда коноид симметричен относительно оси поля, среднее значение коэффициента коррекции рассчитывается при $\theta_{\Pi}=\pi-0.5 \varphi$ и $\theta_{K}=\pi$. Специфика геометрии объемов, в которые попадут электроны после столкновения в зонах 1 и 2 состоит в том, что основания коноида отражения из этих зон составляют не круги, а сложные фигуры пересечения коноида с КО. Приближенно будем считать эти основания эллипсами с полуосями $a$ и $b$, причем углы образующих, которые соединяют концы полуосей с вершиной коноида, обозначим $\varphi_{a}$ и $\varphi_{b}$. Угол при вершине эквивалентного коноида определяется из условия одинаковых объемов реального и эквивалентного коноидов $\varphi_{v}=\operatorname{arctg}\left(\operatorname{tg} \varphi_{b} \sqrt{\operatorname{tg} \varphi_{a} / \operatorname{tg} \varphi_{b}}\right)$. Значения углов для отдельных зон определяются: для зоны 1

$$
\varphi_{q}=2 \operatorname{arctg}(\Delta / \bar{l}), \quad \varphi_{Q}=2 \arcsin (y / r),
$$

$$
\begin{gathered}
\text { где } y=\sqrt{R^{2}-\frac{\left(R^{2}+(R+0.5 r)^{2}-r^{2}\right)^{2}}{4(R+0.5 r)^{2}}}, \\
\varphi_{b q}=0.5 \varphi_{q}, \quad \varphi_{a q}=0.5 \varphi_{Q}, \\
\theta_{\Pi}=0.5\left(\pi-\varphi_{v 1}\right), \quad \theta_{K 1}=0.5\left(\pi+\varphi_{v 1}\right) ;
\end{gathered}
$$

для зоны 2

$$
\begin{gathered}
\varphi_{n q}=\arccos (0.424)-\operatorname{arctg}(0.424 r /(\Delta+0.424 r)), \\
\varphi_{c}=\operatorname{arctg}(0.424 r /(\Delta+0.424 r))+0.5 \varphi_{n q}
\end{gathered}
$$

$\varphi_{\Pi Q}=$

$$
\begin{gathered}
=2 \arcsin \left(\sqrt{R^{2}-\left(\left(R^{2}+(R+0.424 r)^{2}-r^{2} \sin ^{2} \varphi_{e}\right) / 2(R+0.424 r)\right)^{2}} / r\right), \\
\varphi_{b n q}=0.5 \varphi_{n q}, \quad \varphi_{a_{n q}}=0.5 \varphi_{\Pi Q}, \\
\theta_{\Pi 2}=\varphi_{e}-0.5 \varphi_{v 2}, \quad \theta_{K 2}=\varphi_{e}+0.5 \varphi_{v 2} ;
\end{gathered}
$$

для зоны 3

$$
\varphi_{n}=0.667 \pi, \quad \varphi_{v 3}=0.333 \pi,
$$




$$
\theta_{\Pi 3}=\pi-\varphi_{v 3}, \quad \theta_{K 3}=\pi
$$

где обозначения в формулах (16)-(21) $\varphi_{v(1-2)}$ - угол при вершине эквивалентного коноида, который определяется по формуле (16). Поскольку схема столкновения сферически симметричная, вероятность отражения в коноид с углом при вершине $\varphi$ равна отношению телесного угла коноида $\Theta$ к полному телесному углу сферы. Телесный угол определяется, если учесть, что один стерадиан вырезает на поверхности сферы площадь, размер которой $r^{2}$ (рис. 2). Площадь поверхности сферического сегмента $s_{\varphi}=2 \pi r^{2}(1-\cos (\varphi / 2))$, поэтому телесный угол $\Theta=2 \pi(1-\cos (\varphi / 2))$, откуда вероятность отражения в этот телесный угол (принимая во внимание, что $\left(0.5(1-\cos (\varphi / 2))=\sin ^{2}(\varphi / 4)\right)$ $P_{B}(\varphi)=\sin ^{2}(\varphi / 4)$. При этом в качестве угла $\varphi$ для каждой из трех зон принимается значение соответствующего угла при вершине эквивалентного коноида, определяемого по формуле (15). Общая вероятность попадания электронов в КО в результате первичного отражения из всех зон $P_{B}=1-\left(1-P_{B}(1)\right)\left(1-P_{B}(2)\right)\left(1-P_{B}(3)\right)$, а статистическая вероятность наличия в КО электронов с базовой скоростью $V_{x} P_{V_{x}}=1-\left(1-P_{0}\right)\left(1-P_{B}\right)$. Из рис. 3 следует, что одному и тому же значению распределения $M_{x}$ соответствуют две скорости. Очевидно, нужно оперировать большим значением скорости (правая ветвь кривой), поскольку по определению базовая скорость должна находиться в области наибольших из возможных, как скорость, которая сформирована бесстолкновительным движением.

Тогда из формулы (1)

$$
P_{V x}=M_{x} \Delta V=\frac{4}{\sqrt{\pi}} \frac{V_{x}^{2}}{c^{3}} e^{-\frac{V_{x}^{2}}{c^{2}}} \Delta V
$$

Наиболее вероятная скорость электронов в слое определится из выражения $c^{3} e^{\frac{V_{x}^{2}}{c^{2}}}=\frac{4 V_{x}^{2} \Delta V}{P_{V x} \sqrt{\pi}}=\frac{4 K_{V} V_{x}^{3}}{P_{V x} \sqrt{\pi}}=$ $=\frac{2.257 K_{V} V_{x}^{3}}{P_{V x}}$. После преобразования в вид, приемлемый для дальнейшего исследования $3 \ln (c)+\frac{V_{x}^{2}}{c^{2}}-\ln \frac{4 \cdot V_{x}^{2}}{M_{x} \sqrt{\pi}}=0$, получим решение $c=\frac{1}{3}\left[-6 V_{x}^{2} / W\left(\frac{-V_{x}^{2}}{24} \sqrt[3]{\frac{16^{2} \pi}{V_{x}^{4} / M_{x}^{2}}}\right)\right]^{0.5}$, которое после упрощения принимает окончательный вид

$$
c=0.816\left[-V_{x}^{2} / W\left(-0.387 \sqrt[3]{M_{x}^{2} / V_{x}^{4}}\right)\right]^{0.5},
$$

где $W$ - специальная функция Ламберта, которая базируется на решении уравнения типа $x=y e^{y}$ относительно $y$.

Среднее значение скорости электронов соответствует $[3] \bar{V}=1.128 c$ и в окончательном виде

$$
c=0.921\left[-V_{x}^{2} / W\left(-0.387 \sqrt[3]{M_{x}^{2} / V_{x}^{4}}\right)\right]^{0.5} .
$$

\section{Экспериментальная апробация методики расчета средней скорости электронов}

Экспериментальная проверка достоверности предлагаемой методики проводилась путем сравнения расчетных значений электрических параметров разряда с опытными, полученными на промышленной установке для азотирования в тлеющем разряде. Расчет электрических параметров проводился путем определения потока ионов как результата всех субпроцессов в ОКП. Для этого ОКП разбивалась на 50 слоев, для каждого из которых определялся поток ионов и их сток на катод. Общий алгоритм расчета электрических параметров разряда показан на рис. 4. С применением программ, составленных на основе этого алгоритма, получены расчетные значения тока разряда. Аппроксимация распределения средней скорости электронов проводилась, исходя из следующих начальных предпосылок. Средняя скорость электронов после достижения определенного максимального значения $V_{M}$ в некотором слое, который назовем слоем максимума с номером $z_{M}$ и координатой линейного поля $x=z_{M} \delta / 50$, плавно уменьшается и на анодной границе ОКП при энергии $2 \mathrm{eV}$ состав-

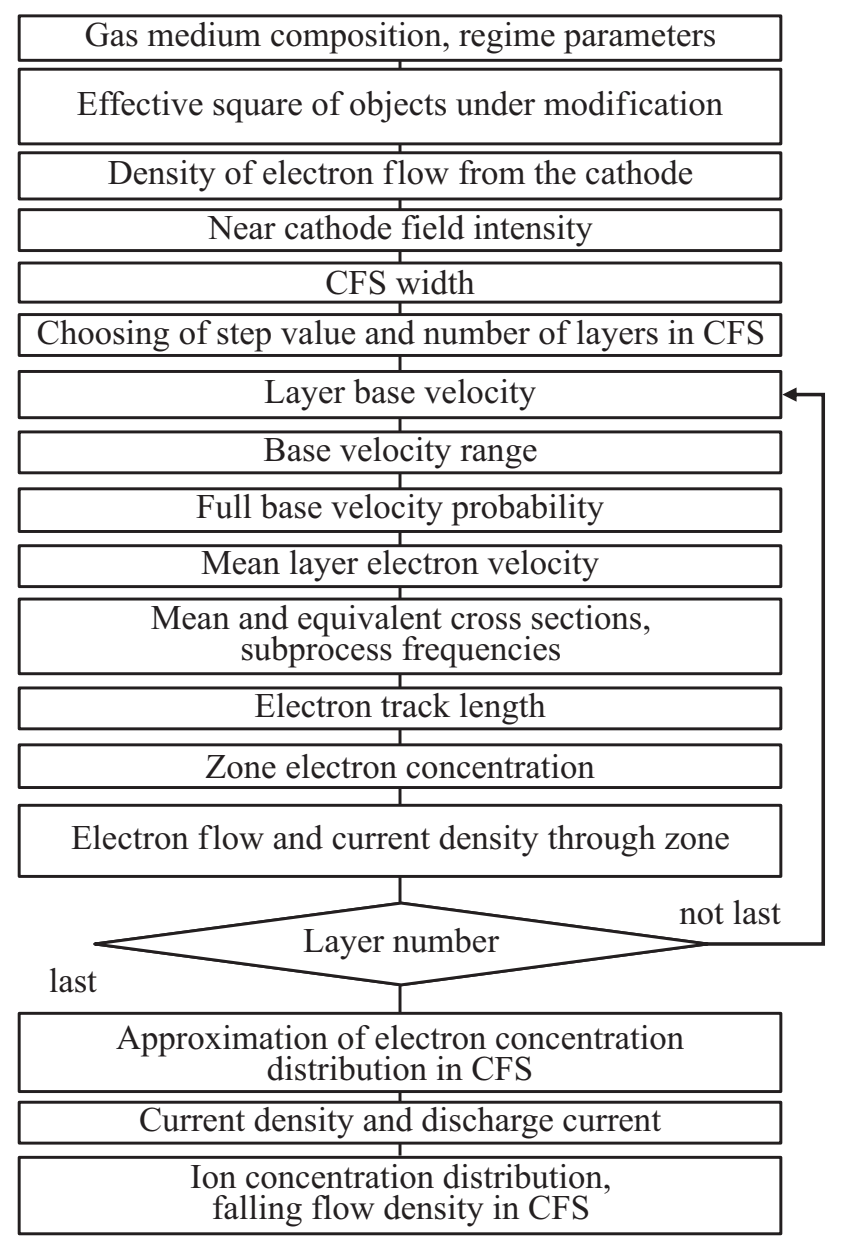

Рис. 4. Алгоритм расчета электрических параметров разряда. 


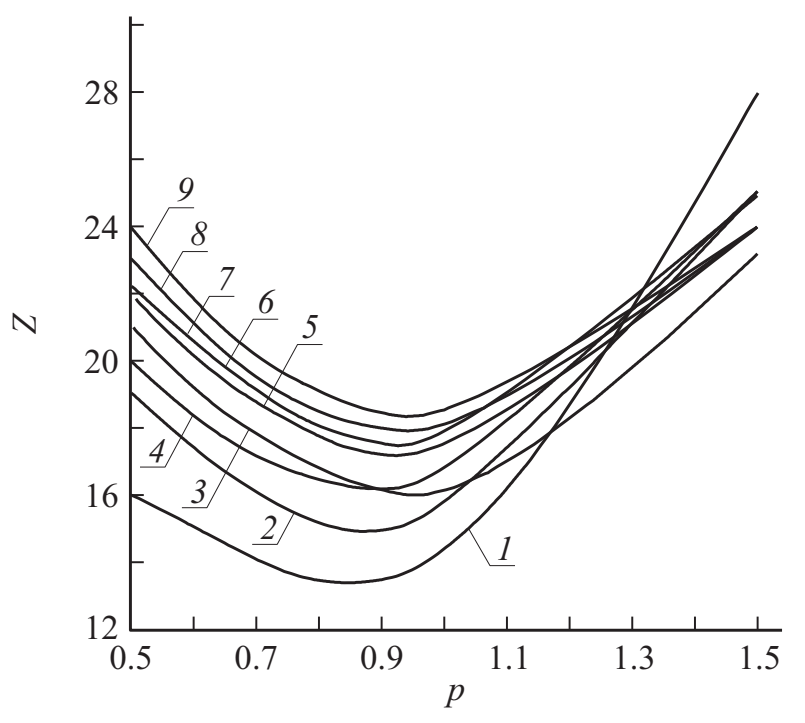

Рис. 5. Изменение номера слоя с максимумом скорости электронов (смесь N75Ar25, $U=300 \mathrm{~V}$ ) при параметрах режима, T: $1-3-400,4-6-500,7-9-600^{\circ} \mathrm{C} ; j: 1-5,2-10$, $3-20,4-12,5-20,6-30,7-20,8-30,9-50 \mathrm{~A} / \mathrm{m}^{2}$.

ляет примерно $V_{K}=0.839 \cdot 10^{6} \mathrm{~m} / \mathrm{s}$ (число электронов с энергией 3-4 eV на два-три порядка меньше [4]). Еще одна опорная точка аппроксимируемой функции изменения средней скорости электронов соответствует поверхности катода, учитывая, что средняя энергия электронов, покидающих катод, примерно равна $1 \mathrm{eV}$, что соответствует скорости $V_{0}=0.5931 \cdot 10^{6} \mathrm{~m} / \mathrm{s}$. Кривая послойного изменения средней скорости электронов на рис. 1 наиболее корректно отображается формулой вида

$$
\bar{V}_{c}(z)=V_{e 0}+a_{V} z e^{-b_{V} c^{c_{V}}} \cdot 10^{6},
$$

где $a_{V}, b_{V}, c_{V}-$ коэффициенты, зависящие от условий, при которых реализуется разряд (состав газовой среды, ее давление, температура газовой компоненты, плотность тока), $z$ - номер слоя ОКП при их общем числе 50, который связан с координатой поля $x$ в направлении от катода формулой $x=0.02 \delta z$.

Расчеты выполнены для большого объема комбинаций параметров режима, причем после достижения нулевого значения вероятности бесстолкновительного движения электронов от катода до контрольного объема дальнейший расчет скорости прекращался. Однако во всех случаях представлялось возможным фиксировать максимальное значение скорости и номер участка, которому соответствовала эта скорость. Влияние параметров технологического режима продемонстрировано на рис. 5 (номер слоя с максимумом скорости) и на рис. 6 (максимальная скорость), $\left(j-\right.$ плотность тока в $\mathrm{A} / \mathrm{m}^{2}$, $T$ - температура газовой среды в градусах Цельсия, $U$ - напряжение в камере $\mathrm{V}$, давление $p$ в Torr, средняя скорость электронов в $\mathrm{m} / \mathrm{s}$ ).

Из рис. 5,6 следует, что экстремумы и номера слоя с максимальной средней скоростью электронов, и самой средней скорости электронов в ОКП соответствуют диапазону давления в разрядной камере от 0.8 до 1 Torr. Наличие этих двух характеристик - максимальной скорости и ее положения в ОКП - с учетом ранее установленных значений средней скорости в крайних точках ОКП позволяет идентифицировать кривую изменения средней скорости электронов по всей ширине ОКП. На рис. 7 представлено сопоставление зависимостей изменения средней скорости по ширине ОКП (соотношение координаты к ширине ОКП).

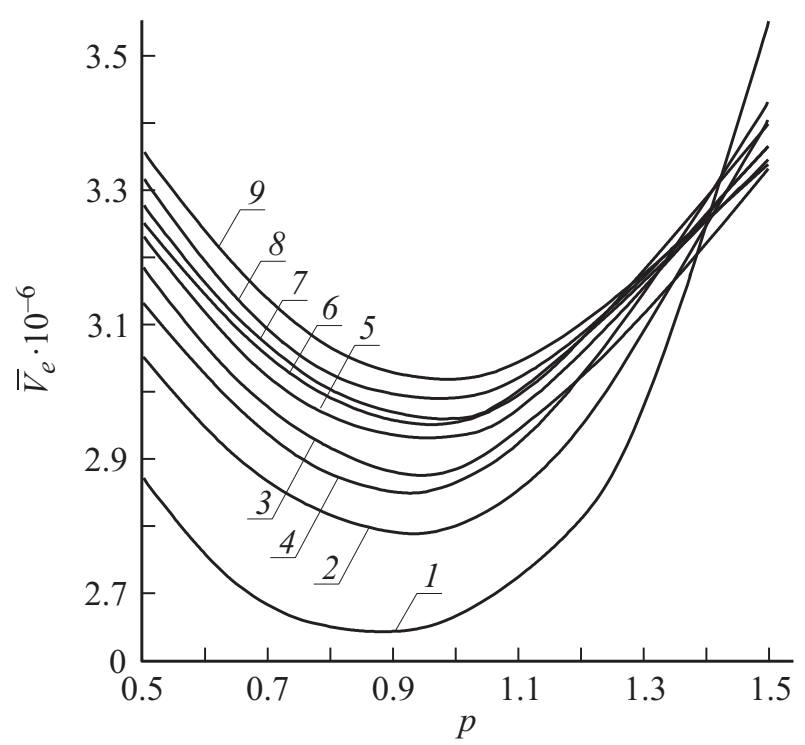

Рис. 6. Изменение максимума средней скорости электронов (смесь N75Ar25, $U=300 \mathrm{~V}$ ) при параметрах режима, $T$ : $1-3-400,4-6-500,7-9-600^{\circ} \mathrm{C} ; j: 1-5,2-10$, $3-20,4-12,5-20,6-30,7-20,8-30,9-$ $50 \mathrm{~A} / \mathrm{m}^{2}$ (давление $p$ в Torr).

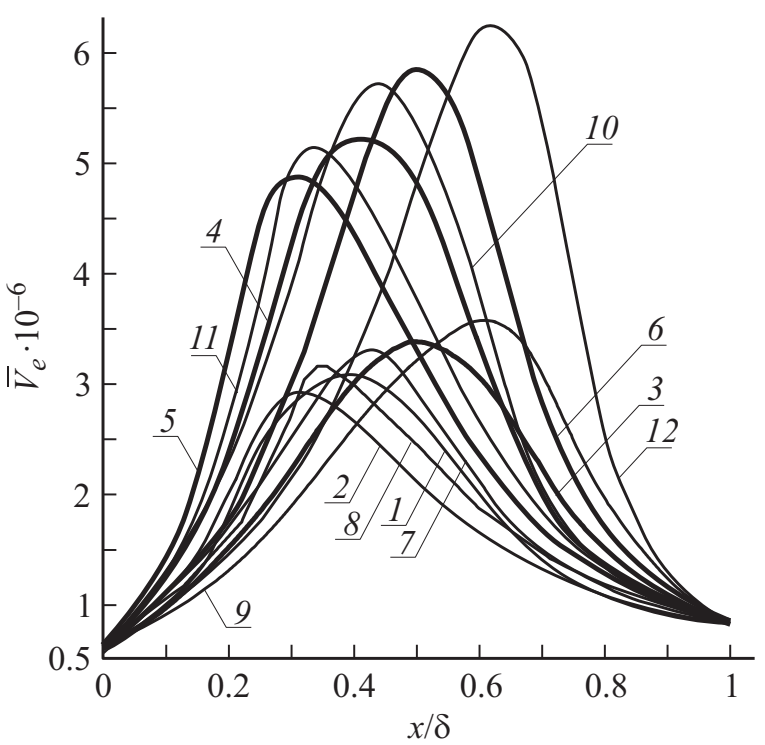

Рис. 7. Изменение средней скорости электронов по ширине ОКП, $U: 1,3,5,7,9,11-300 ; 2,4,6,8,10,12-900 \mathrm{~V} ; p: 1$, $4,7,10-0.5 ; 2,5,8,11-0.9$ Torr; $3,6,9,12-1.5$ Torr. 


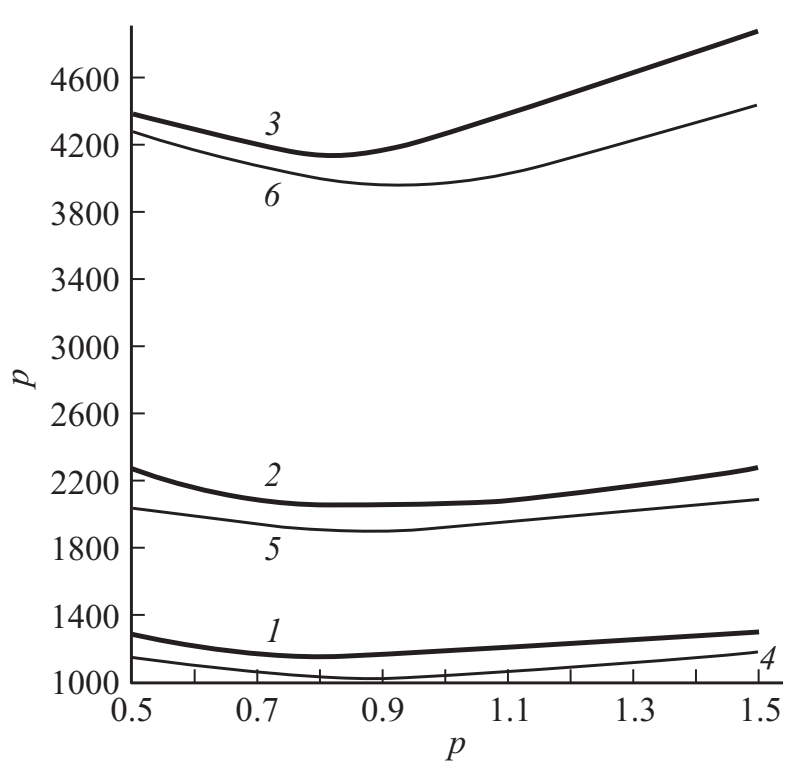

Рис. 8. Зависимость активной (потребляемой) мощности (VA) от давления $p$ (Torr) в режиме термостабилизации (толстые - смесь N75Ar25, тонкие - N10Ar90): 1, 4 - температура $400 ; 2,5-500 ; 3,6-600^{\circ} \mathrm{C}$.

Зависимости приведены для двух составов газовых сред: N75Ar25 - толстые линии и N10Ar90 тонкие линии. Для упрощения информации применены данные только для температуры $500^{\circ} \mathrm{C}$. Рис. 7 подтверждает, что напряжение катодного падения не изменяет положения максимального значения средней скорости электронов, однако влияет на ее величину. Экстремумы на графиках для смеси, в которой преобладает аргон, смещены дальше от катода и максимальная скорость для них выше, что объясняется большей ионизационной активностью аргона, для которого больше значение приведенного интегрального сечения ионизации. Этим же и объясняется экспериментально установленный факт, что для газовых сред с преимущественным содержанием аргона при одинаковых условиях одна и та же плотность тока достигается при меньших значениях напряжения. Выдвинута гипотеза, что наличие минимума проводимости газовой среды может объясняться взаимным перекрытием газокинетических сечений отдельных частиц с увеличением их количества при росте давления или уменьшении температуры. Сначала при увеличении давления (количества контрагентов столкновений с электронами) проводимость газовой среды растет. Затем нейтральные частицы все больше частично или полностью перекрывают друг друга и эффективность увеличения количества контрагентов снижается. Для повышения энергетических свойств разряда необходимо увеличить катодное падение напряжения, т. е. нагрев детали все в большей степени ведется за счет повышения энергии падающего потока, а не за счет количества бомбардирующих частиц.

С целью сопоставления кинетики электронов и электрических характеристик разряда проведена серия экспе- риментов на промышленной установке ИПА-1512 (диаметр камеры $500 \mathrm{~mm}$, ее высота - $1200 \mathrm{~mm}$ ). Образец детали изготовлен из стали 30 , причем он не имел концентраторов поля, активная площадь вместе с подвеской составляла $0.1573 \mathrm{~m}^{2}$. Прежде всего следует заметить, что представленные на рис. 8 графики, отражающие экспериментальные данные, количественно могут быть в точности повторены только в аналогичных условиях, поскольку все параметры фиксировались при стабильной температуре. Стабилизация же ее возможна только при условии теплового баланса, а он, в свою очередь, зависит от многих факторов: площади и массы детали, расстояния от анода, наличия анодного экрана, характера и интенсивности охлаждения и т. П. Поэтому сейчас важны не столько количественные, сколько качественные соотношения. Однако и в этом случае следует обратить внимание на одно важное обстоятельство. Это вопрос измерения мощности разряда, а особенно - удельной мощности. Прежде всего этот показатель не может быть нормативным, поскольку зависит, как указано выше, от индивидуальных особенностей как детали, так и оборудования. Фиксация мощности как произведения напряжения и тока путем измерения их приборами может в некоторых случаях дать погрешность порядка 300\%. Даже когда в блоке питания применялся LC-фильтр, мощность постоянной составляющей в некоторых случаях была на $35 \%$ меньше активной (потребляемой). Практически во всех экспериментах четко зафиксирован минимум потребляемой (активной) мощности при давлении в пределах 0.8-1 Torr (рис. 8). Сопоставление газовых сред, в которых преобладает азот, со смесями, в которых доминировал аргон, приводит к выводам, полностью совпадающими с предыдущими относительно графиков средних скоростей электронов: разряд в смесях, где преобладает аргон, проходит при меньших напряжениях, а мощность в режиме термостабилизации - меньше. Последнее обстоятельство обосновывается большей массой частиц, бомбардирующих поверхность катода, за счет чего поверхность более интенсивно нагревается при сравнительно меньшей плотности падающего потока и его энергии вследствие большого коэффициента энергопередачи [7]. Однако плотность тока в аргоновой среде преимущественно выше, что объясняется повышенной ионизационной активностью аргона (исключение - при температуре $600^{\circ} \mathrm{C}$, когда более существенными и весомыми становятся явления, связанные с термоэлектронной эмиссией).

Таким образом, сопоставление данных по средней скорости электронов и активной мощности разряда подтверждает наличие минимума в области давлений 0.8-1 Torr. Учитывая, что экстремумы этих двух величин полностью совпадают, можно сделать вывод о том, что средняя скорость электронов является именно той характеристикой, которая формирует электрические параметры разряда в газовой среде, поскольку она определяет интенсивность ионизационных процессов. Характер ее изменения в зависимости от параметров 
технологического режима объясняет качественные соотношения энергетических характеристик разряда, их влияние на результаты модификации поверхности и позволяет считать методику расчета средней скорости электронов в ОКП достоверной.

\section{Практическая методика расчета средней скорости электронов}

Тем не менее нельзя не признать, что вычислительный аппарат изложенной выше методики несколько громоздок и требует наличия специального программного продукта. Результаты расчетов для удобства практического применения в исследовательской и технической практике можно получить, используя приведенный ниже алгоритм. Предлагаемая методика определяет коэффициенты уравнения (25) в два этапа: сначала в зависимости от условий, в которых существует электрический разряд в газе, рассчитываются максимальные значения скорости $V_{e M}$ и номер слоя, который соответствует этому максимуму. Затем эти два параметра переводятся в значения коэффициентов уравнения (25). Для газовой среды, которая полностью состоит только из азота (N100), максимальное значение средней скорости электронов в $\mathrm{m} / \mathrm{s}$ как функции давления $p$ (в пределах 0-1.2 Torr), плотности тока $j\left(4-120 \mathrm{~A} / \mathrm{m}^{2}\right)$, температуры газовой компоненты $T(300-873 \mathrm{~K})$, межэлектродного потенциала $U(250-550 \mathrm{~V} \mathrm{~V})$ рассчитывается системой уравнений в последовательности исключения факторов влияния: давления

$$
\left\{\begin{array}{l}
K_{P 1}(p)=1.565-1.415 p+0.85 p^{2}, \\
K_{P 2}(p)=0.075+0.064 p-0.066 p^{2}, \\
K_{P 3}(p)=0.0008601-0.0006156 p+0.0003584 p^{2}, \\
K_{P 4}(p)=-0.245+0.284 p-0.166 p^{2}, \\
K_{P 5}(p)=0.004206-0.003856 p+0.002291 p^{2}, \\
K_{P 6}(p)=0.063+0.089 p-0.078 p^{2}, \\
K_{P 7}(p)=0.000002069-0.000001228 p \\
\\
+0.00000075 p^{2}, \\
K_{P 8}(p)=-0.178+0.134 p-0.084 p^{2},
\end{array}\right.
$$

плотности тока

$$
\left\{\begin{array}{l}
K_{P J 1}(p, j)=K_{P 1}(p) j^{K_{P 2}(p)} \\
K_{P J 2}(p, j)=K_{P 3}(p) j^{K_{P 4}(p)} \\
K_{P J 3}(p, j)=K_{P 5}(p) j^{K_{P 6}(p)} \\
K_{P J 4}(p, j)=K_{P 7}(p) j^{K_{P 8}(p)}
\end{array}\right.
$$

температуры

$$
\left\{\begin{array}{l}
K_{T P J 1}(T, p, j)=K_{P J 1}(p, j)+K_{P J 2}(p, j) T, \\
K_{T P J 2}(T, p, j)=K_{P J 3}(p, j)+K_{P J 4}(p, j) T,
\end{array}\right.
$$

межэлектродного потенциала

$$
\begin{aligned}
\bar{V}_{e M}(U, T, p, j) & =\left(K_{T P J 1}(T, p, j)\right. \\
& \left.+K_{T P J 2}(T, p, j) U\right) \cdot 10^{6} .
\end{aligned}
$$

Аналогичным порядком определяется номер слоя, в котором средняя скорость будет максимальной, однако, как установлено выше, так как положение максимума не зависит от межэлектродного потенциала $U$, количество факторов исключения будет меньше, а именно давление

$$
\left\{\begin{array}{l}
K_{Z P 1}(p)=13.648-19.871 p+11.766 p^{2} \\
K_{Z P 2}(p)=0.167+0.144 p-0.153 p^{2}, \\
K_{Z P 3}(p)=0.021-0.026 p+0.014 p^{2}, \\
K_{Z P 4}(p)=-0.079+0.193 p-0.131 p^{2},
\end{array}\right.
$$

плотность тока

$$
\left\{\begin{array}{l}
K_{Z P J 1}(p, j)=K_{Z P 1}(p) j^{K_{Z P 2}}, \\
K_{Z P J 2}(p, j)=K_{Z P 3}(p) j^{K_{Z P 4}},
\end{array}\right.
$$

температура

$$
z_{M}(T, p, j)=K_{Z P J 1}(p, j)+K_{Z P J 2}(p, j) T
$$

с последующим округлением к ближайшему целому.

Сопоставление результатов расчета максимальной средней скорости электронов и положения максимального значения в ОКП по приведенной выше методике с начальными значениями этих параметров дает отклонение по скорости в пределах $2 \%$, по положению максимума не более одного слоя (согласно формуле (25), ОКП разбивалась на 50 слоев, хотя абсолютное значение количества слоев не имеет принципиального значения), что свидетельствует о ее приемлемости.

Дальнейший переход значений скорости и номера слоя с ее максимумом к коэффициентам $a_{V}, b_{V}, c_{V}$ уравнения (25) в принципе возможен при бесконечном числе комбинаций этих коэффициентов. Однако произвольный выбор хотя бы одного из них может привести к значениям других, которые будут или чрезвычайно большими или, наоборот, очень маленькими, что окажет существенное влияние на точность расчетов. Например, большие значения коэффициента $c_{V}$ требуют очень точного представления коэффициента $b_{V}$ (до 8-10 знаков после запятой) и т. п. Так как коэффициент $a_{V}$ влияет только на величину максимального значения средней скорости, однако не изменяет положения максимума, целесообразно было бы выбор начать именно с него из системы уравнений

$$
\left\{\begin{array}{l}
K_{a 1}\left(z_{M}\right)=1900 z_{M}^{-4.5}-0.002241 \\
K_{a 2}\left(z_{M}\right)=9.581 z_{M}^{-1.652} \\
a_{V}\left(z_{M}, \bar{V}_{e M}\right)=K_{a 1}\left(z_{M}\right)+K_{a 2}\left(z_{M}\right) \bar{V}_{e M} \cdot 10^{-6} .
\end{array}\right.
$$

Тогда, учитывая значение средней скорости электронов в начальной и конечной точках (катодная и соответственно анодная границы ОКП), а также в точке максимума, введя дополнительные обозначения $w=\ln \left(\frac{\bar{V}_{e M}-V_{e 0}}{a_{V} z_{M} \cdot 10^{6}}\right), v=\ln \left(\frac{0.004918}{a_{V}}\right)$, получим выражения для расчета других двух коэффициентов

$$
c_{V}=\frac{\ln w-\ln v}{\ln z_{M}-3.912},
$$




$$
b_{V}=-\frac{v}{50^{c_{V}}}=-\frac{w}{z_{M}^{c_{V}}} .
$$

В конечном итоге сопоставление кривых средней скорости с коэффициентами, рассчитанными по (34), (35), с табличными начальными кривыми, указывает на точность результата не хуже $2 \%$ (отклонение в основном по положению слоя максимума, которое следует из необходимости округления расчетных значений номера слоя к ближайшему целому, при необходимости эту точность можно повысить за счет увеличения числа слоев в ОКП).

Результатом значительного объема расчетов стала разработка методики определения значений средней скорости электронов для произвольного состава газовой среды. Так как само понятие базовой скорости, составляющей основу представленной выше методики, предусматривает в качестве главного фактора влияния на вероятность бесстолкновительного движения электрона газокинетическое сечение частиц газа, то логичным было бы введение термина приведенного газокинетического сечения для произвольной газовой среды

$$
\sigma_{G}=\sum_{1}^{i} K_{p W_{i}} \sigma_{G W_{i}},
$$

где $\sigma_{G w i}-$ газокинетическое сечение компонента газовой среды, для атомарного аргона $-40.2 \cdot 10^{-20} \mathrm{~m}^{2}$, для молекулярного азота - $43.1 \cdot 10^{-20} \mathrm{~m}^{2}$, для молекулярного водорода $-22.9 \cdot 10^{-20} \mathrm{~m}^{2}$ [6], соответственно для наиболее применяемых газовых сред $\mathrm{N} 25 \mathrm{Ar} 75-40.92 \cdot 10^{-20} \mathrm{~m}^{2}, \quad \mathrm{~N} 50 \mathrm{Ar} 50-$ $41.65 \cdot 10^{-20} \mathrm{~m}^{2}, \quad \mathrm{~N} 75 \operatorname{Ar} 25-42.37 \cdot 10^{-20} \mathrm{~m}^{2}$, $\mathrm{N} 90 \mathrm{H} 10-41.08 \cdot 10^{-20} \mathrm{~m}^{2}, \quad \mathrm{~N} 10 \mathrm{Ar} 90 \quad-$ $40.49 \cdot 10^{-20} \mathrm{~m}^{2}$.

Установлена линейная связь между соотношением максимальных значений средних скоростей электронов для произвольных газовых сред и среды чистого азота

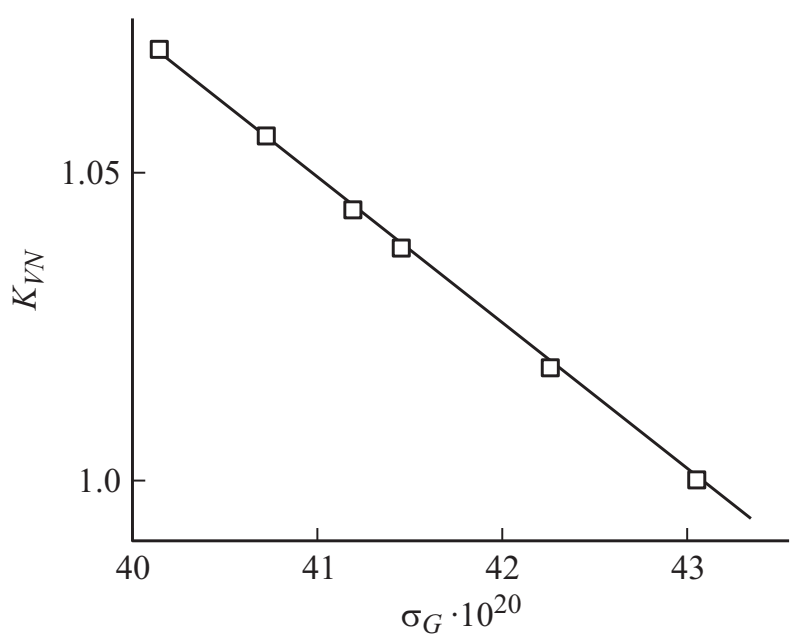

Рис. 9. Взаимосвязь между соотношениями средних скоростей применительно к среде чистого азота и приведенным газокинетическим сечениям среды.

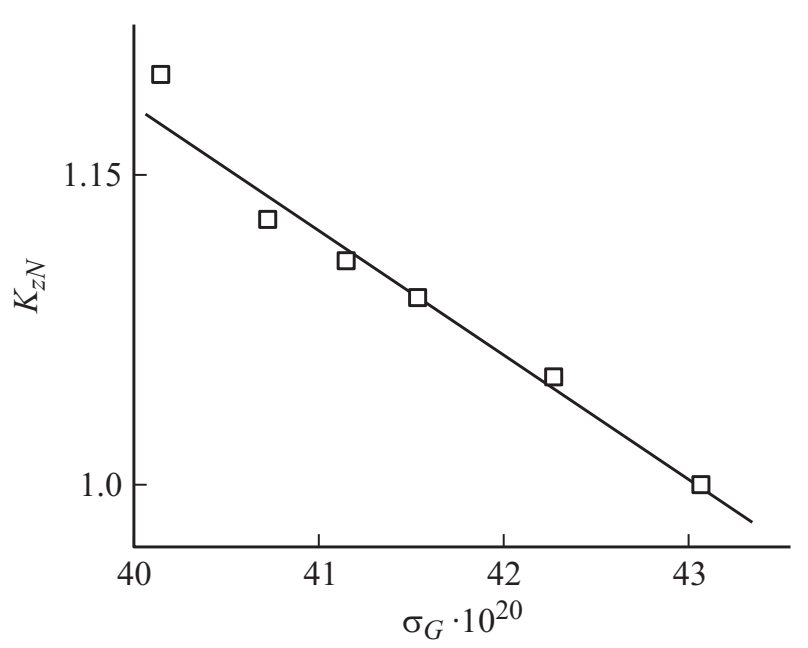

Рис. 10. Взаимосвязь между соотношениями положений средних скоростей применительно к среде чистого азота и приведенным газокинетическим сечениям среды.

(рис. 9), а также аналогичным отношением положений максимумов (рис. 10) и приведенным газокинетическим сечением среды (коэффициенты корреляции соответственно 1 и 0.965$)$.

Таким образом, для произвольной газовой среды максимальное значение средней скорости электронов определяется как произведение максимального значения этой скорости в газовой среде чистого азота на коэффициент влияния приведенного газокинетического сечения $\bar{V}_{e M W}=\bar{V}_{e M} K_{V N}$, аналогично положение максимума средней скорости $z_{M W}=z_{M} K_{z N}$, где из рис. 9 и 10 соответственно

$$
\begin{gathered}
K_{V N}=2.06751-0.024784 \sigma_{G} \cdot 10^{20}, \\
K_{z N}=2.92137-0.44783 \sigma_{G} \cdot 10^{20} .
\end{gathered}
$$

\section{Заключение}

Практически во всех субпроцессах в области катодного падения тлеющего разряда инициатором выступают электроны. Вероятность любого из субпроцессов, определяемая сечением, самым существенным образом зависит от скорости электронов в момент столкновения с частицами газа.

Предложена концепция определения средней скорости электронов на базе модели базовой скорости как скорости, которую имеет электрон, пролетевший без столкновений от катода к определенной точке области катодного падения.

Выполнена экспериментальная проверка методики определения средней скорости электронов на основе сопоставления расчетных и опытных значений электрических параметров разряда.

Создано программное обеспечение для расчетов средней скорости электронов в любой точке катодного падения разряда. 
Обработаны результаты расчетов и на их базе предложена методика практического расчета энергетических характеристик движения электронов.

\section{Список литературы}

[1] Пастух И.М. // ЖТФ. 2016. Т. 86. Вып. 1. С. 78-86.

[2] Пастух И.М. // ЖТФ. 2014. Т. 84. Вып. 9. С. 60-65.

[3] Мирдель Г. Электрофизика: Пер. с нем. / Под ред. В.И. Рахновского. М.: Мир, 1972. 608 с.

[4] Райзер Ю.П. Основы современной физики газоразрядных процессов. М.: Наука, 1980. 416 с.

[5] Druyvesteyn M.I., Penning F.M. Mechanism of Electrical Discharge in Gases of Low Pressure. Rev. Mod. Phys. 1940. V. 12.

[6] Райзер Ю.П. Физика газового разряда. М.: Наука, 1987. $592 \mathrm{c}$.

[7] Пастух И.М. // ЖТФ. 2013. Т. 83. Вып. 8. С. 144-147. 